\title{
LA FICHA CLÍNICA MIRADA DESDE LA LEGISLACIÓN CHILENA ACTUAL
}

\author{
Jorge Godoy Olave', Jaime Barraza Mesquida²
}

Resumen: La historia o ficha clínica ha servido de instrumento de registro de las actividades sanitarias desde el inicio de las profesiones de salud, otorgándosele diversas utilidades y valoraciones, según su objetivo, un valor docente, en investigación, judicial, entre otros. Ante las diferentes interpretaciones de las normas vigentes en Chile se ha debido legislar en su uso, pertenencia, contenido y otros aspectos, aclarando algunas situaciones pero restringiendo su acceso en otros aspectos. Dado las distintas leyes y normativas a las que se asocia su uso, se hizo necesario elaborar un documento que incluya sus aspectos más importantes. Aún quedan elementos asociados a las costumbres, creencias, especialidades en salud, entre otras, que no han sido abordados por las leyes, además de la labor docente de este instrumento.

Palabras clave: historia médica, legislación, jurisprudencia, derechos del paciente, consentimiento informado

\section{Clinical record looked at from the current Chilean legislation}

Abstract: The history or clinical record has served as an element of registration of health activities since the beginning of the health professions, granting him other utilities and valuations according to their objective, as a teaching value, in research, judicial, among others. Given the different interpretations of the norms in force in Chile, it has had to legislate in its use, membership, content and other aspects of the clinical file, clarifying some situations, but restricting their access in other aspects. Given the different laws and regulations associated with its use, it became necessary to produce a document that collects its most important aspects. There are still aspects associated with customs, beliefs, specialties in health, among others, that have not been addressed by the Laws, in addition to the teaching work of this instrument.

Key words: medical record, legislation, jurisprudence, patient rights, informed consent

\section{A ficha clínica vista a partir da legislaçáo chilena atual}

Resumo: A história ou ficha clínica tem servido como instrumento de registro das atividades sanitárias desde o início das profissóes de saúde, concedendo-se a elas vários utilitários e valoraçóes de acordo com sua finalidade: ensino, pesquisa, valor jurídico, entre outros. Para as diferentes interpretações das normas vigentes no Chile havia de legislar em seu uso, composição, conteúdo e outros aspectos da ficha clínica, esclarecendo algumas situaçóes, mas restringindo seu acesso em outros aspectos. Tendo em conta as diferentes leis e regulamentos aos quais o seu uso está associado, tornou-se necessário apresentar um documento que reúne os aspectos mais importantes. Existem ainda os aspectos associados a costumes, crenças, especialidades em saúde, entre outros, que não foram abordadas pelas leis, além do ensino deste instrumento.

Palavras-chave: história médica, legislação, jurisprudência, direitos do paciente, consentimento informado

\footnotetext{
${ }^{1}$ Facultad de Odontología, Universidad de Valparaíso, Chile

Correspondencia: jorge.godoy@uv.cl

${ }^{2}$ Universidad de Valparaíso, Chile
} 


\section{Introducción}

En la historia de las actividades sanitarias siempre ha sido central el registro de las atenciones( 1 , 2), siendo la historia clínica una guía para todo el quehacer sanitario, estadístico, investigativo, entre otros. El Código Sanitario de 1931 considera estos datos en su Libro I, Título IV: de las estadísticas sanitarias. Y en el Libro VI: de los Establecimientos del Área de la Salud, en su artículo 134, se habla de la presencia de los registros, libros, fichas clínicas y documentos, considerados relevantes para resguardar la información y para la toma de decisiones en las actividades sanitarias. Desde el inicio del Sistema Nacional de Salud, en 1952, se usa en los hospitales públicos la ficha clínica, en forma de hojas en orden cronológico. Esto ha sido una inquietud no solo nacional. Como ejemplo tenemos la Ley 41/2002, de la Jefatura del Estado Español, reguladora de la autonomía del paciente y de derechos y obligaciones en materia de información y documentación clínica(3). En la República de Colombia se dicta la Resolución 1995 del Ministerio de Salud y Protección Social, en 1999, la cual establece normas para el manejo de la historia clínica(4).

En el año 2000, bajo el mandato del presidente Ricardo Lagos Escobar, se establece una Comisión de Estudios para la Reforma en Salud Chilena, obedeciendo a los cambios sustanciales en este ámbito en América Latina y en el Caribe. En ese periodo la región se encontraba en una reorganización de los Estados, basando sus esfuerzos en el sector salud y la seguridad social, para así mantener la salud de la población, prolongar la vida y reducir las desigualdades. Es así como, fruto de su trabajo, surge un conjunto de cinco proyectos legislativos enviados al Congreso en mayo de 2002: la Ley del Financiamiento en Salud(5); la Ley del Régimen de Garantías Explícitas en Salud(6); la Ley de ISAPRE(7); la Ley de la Autoridad Sanitaria y Gestión(8) y la Ley de Derechos y Deberes de los Pacientes(9). Estas leyes son la base jurídica de la Reforma en Salud, que se centra en la persona con un enfoque familiar y comunitario. Ante esto, la protección de la privacidad y la información, y de los derechos del paciente ha cobrado vital importancia, evidenciándose ello en la ficha clínica.
Ante este escenario, el objetivo de esta publicación es reunir la información sobre esta herramienta clínica, bajo la mirada de la legislación actual y según el valor asignado a ella, dependiendo de la utilidad que se le quiera otorgar.

La Ficha Clínica, antes denominada historia o historial clínico, es aquel documento en el que se registran los antecedentes relacionados con la salud de las personas(10). Debe ser capaz de informar acerca de la historia biológica de una persona de forma ordenada y cronológica, redactada por el profesional de la salud que realiza la evaluación, pero principalmente registrar las consultas y tratamientos por una enfermedad. En tanto instrumento de registro de información, la ficha tiene valor para la investigación, utilizando su contenido en pos de resolver problemas de salud con metodología científica; valor judicial, ya que es utilizada por abogados y médicos legistas como "documento médico" de prueba ante la justicia, herramienta médico legal; valor institucional, ya que registra las acciones en el paciente dando a conocer el grado de avance en el tratamiento planificado; valor para los tratantes, por convertirse en una herramienta de comunicación entre ellos, y valor para el paciente, por concentrar la historia de sus enfermedades. Se puede agregar un valor docente, en el que el profesional y sus estudiantes comparten la información y desarrollan su aprendizaje con base en el servicio. En la actualidad se le otorga también un valor en el control de calidad de la atención y de los establecimientos de salud, asociado a la gestión y administración de los mismos.

La ficha clínica se considera hoy un instrumento obligatorio para integrar la información del paciente en la atención en salud. Puede llevarse de forma digital/electrónica, en papel u otro medio, debiendo ser registrado su contenido solo por el profesional de salud que otorga la prestación, ya sea en la modalidad de prestador individual o institucional, en el ámbito público o privado. Cada establecimiento o institución posee su propia herramienta de registro, según sus exigencias o especialidades en que se desempeña.

Desde el punto de vista de la Ley de Protección de la Vida Privada, Ley 19.628, en la ficha clínica se consideran como datos sensibles "aquellos 
datos personales que se refieren a las características físicas o morales de las personas o a hechos o circunstancias de su vida privada o intimidad, tales como los hábitos personales, el origen racial, las ideologías y opiniones políticas, las creencias o convicciones religiosas, los estados de salud físicos o psíquicos y la vida sexual"(11). Como explicita esta ley, estos datos no pueden ser objeto de tratamiento, salvo que exista el consentimiento del titular o sean necesarios para la determinación u otorgamiento de beneficios de salud hacia el paciente. Por su parte, las recetas médicas y los exámenes de laboratorios clínicos y servicios, muchas veces anexas a las fichas clínicas, son de carácter reservado. Solo podrá darse a conocer su contenido, o entregar copia de ellos, con el consentimiento escrito expreso del paciente, a menos que se consideren para datos estadísticos respecto de ventas o utilización de medicamentos, sin proporcionar el nombre de los pacientes destinatarios de las recetas, médicos que prescriben los medicamentos u otro dato que permita su identificación.

La implementación de la Ley que "Regula los Derechos y Deberes que tienen las personas en relación con acciones vinculadas a su atención en Salud", Ley 20.584, hizo necesario legislar en el tema de la ficha clínica(9). Esta Ley explicita el derecho de los pacientes a que quede constancia de la atención recibida en salud por el profesional que la otorga, debiendo obligatoriamente ser él quien llene este registro, de forma clara y legible, con un orden secuencial, incluyendo su identificación y su firma, durante o inmediatamente después de la atención realizada.

No toda la información debe ser escrita en la ficha clínica, ya que un grupo de indicaciones proveniente de su atención puede darse solo verbalmente, procurando dejar constancia en la ficha respectiva de haber dado esta información al paciente(10).

Una institución no está obligada a tener una sola ficha clínica de un paciente; cada servicio clínico o unidad de especialidad puede tener su registro propio, pero debiendo consignar en la ficha central institucional, como mínimo, la fecha de atención, el profesional que realizó la prestación, el diagnóstico de la evaluación y los medicamentos prescritos. Respecto de los fármacos, se debe detallar la dosis indicada y los plazos de su administración(10).

Este instrumento de registro debe conservarse de forma tal de garantizar su acceso expedito, al menos por 15 años desde la fecha del último regis$\operatorname{tro}(10)$.

\section{Información que debe contener la ficha clínica}

La ficha o historia clínica es el reflejo del método clínico, un conjunto de procedimientos ordenados que lleva a conseguir un diagnóstico, pronóstico y tratamiento para el paciente. La entrevista médica debe considerar decodificar los mensajes manifestados por el paciente, por lo que requiere determinada capacidad de observación y de discriminación en cuanto a la importancia de lo relatado. Alguna veces, por desconocimiento, la información brindada es incompleta, lo que lleva a los profesionales a indagar respecto a lo ocurrido(12). Por ello, depende de la preparación y del nivel de conocimiento del personal la exhaustiva indagación de la información y el correcto llenado de la ficha clínica.

Toda ficha clínica, en su elaboración y contenido, debe cuidar de contar con la siguiente información $(10,13)$ :

Identificación del paciente: nombre completo del paciente; número y tipo de documento de identificación, como pasaporte, cédula de identidad u otro; sexo; fecha de nacimiento; domicilio; teléfono y/o correo electrónico de contacto; ocupación, y sistema de salud al que pertenece. En caso que el paciente no pueda recibir la información directamente o padezca de dificultades de entendimiento o alteración de su conciencia, se debe registrar el nombre completo del apoderado, cuidador y/o representante legal(9). En cada nueva atención deben confirmarse los antecedentes de identificación de la ficha clínica del paciente, modificando aquellos datos que hayan sido cambiados.

Identificación para la institución: número identificador de la ficha clínica; fecha de la creación; nombre o denominación completa del prestador respectivo con su rol único tributario o cédula de identidad, dependiendo del caso. 
Registro de las atenciones: deben ser anotadas con fecha y por orden cronológico todas las consultas, anamnesis, evoluciones clínicas, indicaciones, procedimientos diagnósticos y/o terapéuticos, intervenciones quirúrgicas, protocolos operatorios y/o quirúrgicos, resultados de exámenes realizados, derivaciones o interconsultas, hojas de evolución clínica, hoja de enfermería, epicrisis u otra información relacionada a la atención prestada. Todo examen u hoja anexada debe registrar el número de la ficha, ya sea escrito o electrónico, de forma de permitir su identificación ante riesgo de pérdida. Un error común es no detallar la indicación de exámenes complementarios ni sus resultados, alterando la continuidad de la historia del paciente.

Decisiones del paciente respecto a su atención: se debe consignar el consentimiento informado de la atención y/o tratamiento, rechazos de tratamientos, solicitud de alta voluntaria, alta disciplinaria y cualquier solicitud del paciente respecto de sus convicciones étnicas, culturales y/o religiosas.

Es común que este instrumento resuma, desde el punto de vista biomédico, lo ocurrido en el paciente, descuidando los aspectos éticos, sociales, culturales, espirituales y psicológicos del mismo. Hay un conjunto de datos posiblemente relevantes que deberían consignarse en la ficha clínica, como los hábitos, costumbres, profesiones, entre otros; más aún con el aumento de la migraciones en la actualidad, que reflejan lo heterogéneo de los usuarios y usuarias. La pesquisa de determinantes sociales de la salud aporta información acerca de factores asociados al problema de salud y a su interacción.

\section{Propiedad de la ficha clínica}

La ficha debe ser gestionada de forma centralizada por la institución o los prestadores individuales en donde reciba la atención el paciente, cuidándose el control en su acceso solo a aquellas personas que puedan tomar conocimiento de su contenido y/o registrar nuevos datos en ella. Se debe llevar un registro detallado de las personas que han accedido a la ficha, así como de las fechas.

En todo momento se debe cuidar la confidenciali- dad de este instrumento, incluso resguardando la ficha del personal de salud $y / o$ administrativo que no tenga relación directa con la prestación $(10,14)$.

Ante esto, la ficha pertenece al paciente, pasando los centros de salud, públicos o privados, y los prestadores individuales a ser solo administradores de su uso. El paciente deberá tener acceso a ella, pudiendo extenderse esta atribución a:

El representante legal del paciente titular de la ficha. En caso de fallecimiento del paciente, esta atribución puede ser otorgada a los herederos y/o herederas del mismo.

Terceras personas, siempre y cuando cuenten con autorización notarial simple.

Tribunales de justicia, si la información a indagar tiene relación con alguna causa que se lleve.

Fiscales del Ministerio Púbico y/o abogados defensores, si cuentan con la autorización oficial de un juez competente, y si la información a indagar tiene relación con alguna causa que se lleve.

No se explicita el permiso a los propios servicios de salud o autoridades competentes, como el Instituto de Salud Pública (ISP), secretarías ministeriales regionales de salud y Superintendencia de Salud cuando se requiera su uso, como ante el estudio de casos sospechosos de alguna condición de impacto en la salud pública o epidemiológica, investigación de información en estudio de brotes u otras condiciones especiales, aunque se reconoce su función e importancia epidemiológica frente a las instituciones(9). El Oficio Ord. $\mathrm{N}^{\circ}$ A15 3392 del Ministerio de Salud, de 29 de octubre de 2012, reitera la necesidad de dotar a determinadas entidades con la función de revisión o estudio de la fichas clínicas, con fines de fiscalizar las prestaciones, determinar beneficios de salud a las personas o para protección de la salud de la población(15). Se ha prohibido a aquellas entidades públicas o privadas vender, ceder o transferir, a cualquier título, las bases de datos que contengan información sensible respecto de sus pacientes, salvo que se trate del otorgamiento de los beneficios de salud que les correspondan u objetivos legales, para lo cual no se requerirá de dicho consentimiento(16). 
Asimismo, se limita el campo de la investigación con datos de las fichas, ya sea por su registro o los exámenes complementarios anexados a ella.

\section{Ficha clínica electrónica}

Es una herramienta que permite acceder a datos del paciente, su historial médico y/o exámenes complementarios. Entre sus principales ventajas destacan la integración de la información, evitar la duplicidad e inconsistencia de esta información, y acceder a una alta disponibilidad de datos(17). Esta facilidad de acceder a los datos podría ser su mayor debilidad, en pro del resguardo de la confidencialidad.

Habitualmente hay dos áreas de ingreso, el área clínica, en la que el profesional de la salud consigna la historia médica en este formato electrónico y accede rápidamente a atenciones anteriores, prescripciones y exámenes complementarios; y el área administrativa-gerencial, que accede a la información para el control de gastos, administración de recursos, entre otras labores(18). La mayoría de estos sistema se basa en roles para gestionar su acceso, siendo poco detallados.

El Decreto 41 del Ministerio de Salud, de 2012, aclara en su artículo 2 que este registro puede ser electrónico(10). Es específico para el soporte electrónico que:

La información se respalde en cada proceso de incorporación de los documentos.

Haya una copia de seguridad en el lugar de operación de los sistemas de información, y otra en un centro de almacenamiento de datos electrónicos que tenga un estricto control de acceso, registro de entrada y salida de respaldos.

Se cuente con medidas de seguridad y barreras de protección frente a accesos no autorizados.

La sustitución de la información por la versión más reciente que se disponga sea en el menor tiempo posible, en casos de alteración no programada.

Los programas permitan la restauración del servicio en el menor tiempo posible en los casos que deje de operar.

\section{Consentimiento informado}

Según la Ley 20.584, sobre los Derechos y Deberes de los Pacientes, todas la personas tienen el derecho de otorgar o denegar su voluntad para ser sometido a algún procedimiento o tratamiento, de forma libre y voluntaria, luego que el profesional le entregue toda la información relacionada(9). Se señala que la información entregada por el profesional debe ser de forma verbal, pero debe quedar constancia de este consentimiento de forma escrita, en la ficha clínica, en caso de intervenciones quirúrgicas, procedimientos diagnósticos o terapéuticos invasivos, o para la aplicación de cualquier procedimiento que conlleve un riesgo relevante y conocido para el paciente. Se excusan de este consentimiento escrito las personas en las que la falta de aplicación del procedimiento suponga un riesgo para la salud pública; cuando su condición de salud o cuadro clínico implique riesgo vital o secuela funcional grave si no se realiza la atención inmediata, y si el paciente no puede expresar su voluntad y su representante legal no se encuentre; o si la persona se encuentra en incapacidad de manifestar su voluntad y su representante legal no exista. En estos casos se deberán adoptar medidas para garantizar la protección de la vida.

Chile ha suscrito en 1990 lo explicitado en la Convención de los Derechos del Niño, dándole a los menores que estén en condiciones de formarse un juicio propio el derecho a su autonomía gradual, debiendo solicitar su asentimiento al realizar cualquier prestación en salud, en función a su edad y madurez(19).

Por su parte el Código de Ética del Colegio Médico de Chile, en el título III sobre las relaciones del médico con sus pacientes, destaca el secreto profesional (20). Menciona que debe guardarse confidencialidad de la información verbal obtenida del paciente y de los registros, debiendo solicitar el consentimiento del paciente para trasmitir dicha información si se requiere la asistencia de otro u otra profesional. Este secreto profesional se debe mantener incluso al cesar los servicios médicos o se encuentre fallecido el paciente. Solo puede vulnerarse en caso de enfermedades de notificación obligatorias (ENO), solicitud de los tribunales de justicia, ante su requerimiento para certificados 
de nacimiento o defunción, si es imprescindible ante el perjuicio grave para el paciente o terceros, o si es necesario para su defensa ante tribunales. El Colegio de Cirujanos Dentistas ingresa el mismo concepto en su título I sobre disposiciones generales, de forma más sucinta, denominando "confidente" a quien le otorgue la información.

Con respecto a las ENO -Decreto 158 del MINSAL_, es obligatorio notificar la enfermedad junto con la identificación del paciente, incluyendo nombres, apellidos, RUT, ficha clínica, domicilio, teléfono, sexo y datos de la historia clínica(21). Solo en el caso de las enfermedades de transmisión sexual se reemplaza el nombre y apellido por el RUT, y el domicilio por la comuna de residencia.

\section{Uso en investigación}

En Chile, la investigación científica biomédica en seres humanos incluye no solo el uso de material humano, sino además la información disponible identificable. El uso del contenido de esta ficha clínica para fines de investigación, repositorio principal de la información del paciente, requiere de la aprobación de un Comité de Ética Científico acreditado y de la autorización del director del establecimiento, quien es el custodio de las fichas clínicas.

El enfoque de derecho de las personas, con respeto a su autonomía y protección de los datos personales, ha llevado a múltiples inquietudes e interpretaciones que han venido aclarándose, con el paso del tiempo, por una comisión asesora del Ministerio de Salud(22). La Ley 20.120, sobre la investigación en el ser humano, genoma y clonación, explicita el consentimiento previo, expreso, libre e informado, o de su representante, mediante la firma de un acta, para ser parte de una investigación(23). Esta acta debe contener, de forma comprensible, la finalidad de la investigación, beneficios, riesgos y procedimientos; debe ser firmada no solo por el paciente, como en el caso del consentimiento de procedimiento clínico, sino además por el responsable de la institución y por el director de la institución en la que se realiza la investigación, como ministro de fe. Este consentimiento puede ser revocado en cualquier momento y por cualquier medio. La ley obliga además a demostrar la idoneidad del investigador, sancionando con la suspensión por tres años del ejercicio profesional a quienes no cumplan con las autorizaciones exigidas, y con la prohibición absoluta de ejercer en el territorio nacional en el caso de reincidencia.

\section{Uso en docencia}

En Chile, desde sus inicios, se ha relacionado la formación en las área de la salud con los centros hospitalarios, utilizando la ficha clínica como una herramienta de la enseńanza dentro de esta relación docente asistencial.

Es así como la ficha clínica ha pasado a ser el registro de actividades de internos y residentes en las distintas especialidades, sirviendo además de elemento de comunicación entre las diversas disciplinas. Es más, ha servido de material para el estudio de los casos, desde un punto de vista clínico y epidemiológico, por lo que la difusión de técnicas y tratamiento se obtenían directamente de las fichas.

Bajo la mirada de la legislación actual, el acceso está restringido, por lo que se hace difícil obtener los datos como ejemplos para la docencia, y su acceso a los estudiantes. Este acceso debe ser permitido por el propio paciente, bajo el marco del consentimiento informado, y su publicación custodiado por el Comité Ético Científico(23).

En cuanto el uso de la ficha clínica con fines no asistenciales, como docencia e investigación, especialmente en estudios retrospectivos, obtener un consentimiento expreso del titular de los datos es casi impracticable, haciendo inviable la investigación. Ante esto, la Comisión Ministerial de Ética de la Investigación en Salud (CMEIS) ha realizado recomendaciones para que los comités de ética científica autoricen el uso de fichas clínicas en estudios sin la obtención de un consentimiento informado por parte del titular

\section{Consideraciones finales}

Queda claro que la legislación considera a la ficha clínica como un "instrumento obligatorio" en la atención en salud, y que su información se considera "dato sensible", por lo que no se puede utili- 
zar a menos que la ley lo autorice. Su utilización se basa en principios como la pertinencia, transparencia, autonomía, responsabilidad, protección universal, confidencialidad, entre otros(24).

En este documento legal debe quedar manifiesto los principios de "no-maleficencia" y "beneficencia" del acto médico, dejando claro que lo aplicado en los usuarios y las usuarias no es caridad, sino la forma de maximizar los beneficios y minimizar los riesgos, respetando su "autonomía". Esta voluntad autorreguladora, manifiesta por parte del paciente, revela su capacidad de actuar a conciencia, con conocimiento de causa y sin coacción externa, con una responsabilidad compartida, como lo demuestra el modelo deliberativo. Estos principios éticos — "justicia" y "no-maleficencia"-, conocidos como "deberes perfectos", son la representación de lo correcto y lo incorrecto, objeto del ámbito de Derecho.

Sus fines son diversos, destacándose su importancia médico-legal, dado que el contenido de la ficha clínica es de valor probatorio en la identificación de víctimas carbonizadas, descompuestas, esqueletizadas, mutiladas y/o fragmentadas(12). Gracias a la estabilidad de la evidencia dental, es la tercera en importancia en el proceso de identificación, siendo superada solo por las huellas digitales y el ADN. Actualmente su rol docente está en entredicho debido a distintas interpretaciones de las normas legales vigentes, lo que hace necesario una revisión o análisis, como lo realizó la Comisión Ministerial de Ética de la Investigación en Salud.

No podemos dejar de mencionar que este acto médico es el encuentro entre dos personas de diferentes realidades y biografías individuales diversas: el tratante con voluntad de ayuda técnica y un usuario con voluntad de curación. La obligación de cuidado del tratante debe encerrar la discreción en el ámbito público, la confidencialidad en el ámbito privado y la dignidad en el ámbito íntimo.

Independiente de lo criticable de las normas y la legislación vigente sobre la ficha clínica, dado su algo grado restrictivo, estas son herramientas para valorar los principios bioéticos en la relación clínica. Es un marco regulador homogéneo tratando de enfatizar el principio de "justicia" y los derechos de los usuarios.

Los autores participaron en la idea, elaboración del manuscrito y en su revisión final. Declaran además no poseer conflictos éticos con el tema, ni con lo enviado en el manuscrito, ni con las instituciones donde se desempeñan.

\section{Referencias}

1. Foucault M. La incorporación del hospital en la tecnología moderna. In: Gabilondo Á, editor. Estética, ética y hermenéutica. Barcelona: Editorial Paidós Ibérica, S.A.; 1999: 97-110.

2. Fombella Posada M, Cereijo Quinteiro M. Historia de la historia clínica. Galicia Clin. 2012; 73(1): 21-26.

3. Sarrato L. El régimen legal de acceso a la historia clínica y sus garantías. Rev Jur de Castilla y León 2009; (17): 177-215.

4. Guzmán F, Arias CA. La historia clínica: elemento fundamental del acto médico. Rev Colomb Cir. 2012; 27(1): 15-24.

5. Ministerio de Hacienda. Ley 19888. Establece el financiamiento necesario para asegurar los objetivos sociales prioritarios del Gobierno. (Publicada en Diario Oficial el 13 de agosto del 2003). Disponible en https://www.leychile.cl/ $\mathrm{N} ? \mathrm{i}=213493 \& \mathrm{f}=2006-04-28 \& \mathrm{p}=$.

6. Ministerio de Salud. Ley 19966. Establece un régimen de garantías en salud (Plan AUGE). (Publicada en Diario Oficial el 03 de septiembre del 2004). Disponible en https://www.leychile.cl/N?i=229834\&f=2004-09-03\&p=.

7. Ministerio de Salud. Ley 20015. Modifica la Ley $N^{\circ} 18.933$ sobre instituciones de salud previsional. (Publicada en Diario Oficial el 17 de mayo del 2005). Disponible en https://www.leychile.cl/N?i=238102\&f=2005-05-17\&p=.

8. Ministerio de Salud. Ley 19937. Modifica el Decreto Ley No 2.763 de 1979 con la finalidad de establecer una nueva concepción de la autoridad sanitaria distintas modalidades de gestión y fortalecer la participación ciudadana. (Publicada en Diario Oficial el 24 de febrero del 2004). Disponible en https://www.leychile.cl/N?i=221629\&f=2008-12-31\&p=.

9. Ministerio de Salud; Subsecretaria de Salud Pública. Ley 20584. Regula los Derechos y Deberes que tienen las personas en relación con acciones vinculadas a su atención en Salud. (Publicada en Diario Oficial el 24 de abril del 2012). Disponible en https:/www.leychile.cl/N?i=1039348\&f=2012-10-01\&p=. 
10. Ministerio de Salud; Subsecretaría de Redes Asistenciales. Decreto 41. Aprueba reglamento sobre fichas clínicas. (Publicada en Diario Oficial el 15 de diciembre del 2012). Disponible en http://www.leychile.cl/N?i=1046753\&f=2012-12$15 \& \mathrm{p}=$.

11. Ministerio Secretaría General de la Presidencia. Ley 19628. Sobre protección de la vida privada. (Publicada en Diario Oficial el 28 de agosto de 1999). Disponible en https://www.leychile.cl/N?i=141599\&f=2012-02-17\&p=.

12. Cuenca K, Rodríguez ML, Soto AD, Pentón O. La historia clínica estomatológica como herramienta en el método clínico y documento médico-legal. Revista Cubana de Medicina Militar 2014; 43(4): 534-540.

13. Alcaraz M, Nápoles Y, Chaveco I, Martínez M, Coello JM. La historia clínica: un documento básico para el personal médico. MEDISAN 2010; 14(7): 1017-1023.

14. Varas J, Hering E, Demetrio AM. Ficha Clínica: - Composición y manejo - Registros clínicos. Revista Obstetricia Ginecología, Hospital Santiago Oriente Dr Luis Tisné Brousse. 2010; 5(1): 57-61.

15. Ramos P, Arenas A. Chile. Acceso a la ficha clínica para investigación científica. Revista Chilena de Derecho 2013; 40(3): 1055-1071.

16. Ministerio de Salud; Subsecretaria de Salud Pública. Ley 20635. Adecua el Decreto con Fuerza de Ley $N^{\circ} 1$, del 2006, del Ministerio de Salud, a la Ley $N^{\circ} 20.575$ que establece el prinicipio de finalidad en el tratamiento de datos personales. (Publicado en el Diario Oficial el 17 de noviembre del 2012). Disponible en https://www.leychile.cl/N?i=1045822\&f=2012$11-17 \& \mathrm{p}=$.

17. Carrion I, Fernandez JL, Toval A. Gestión del control de acceso en historiales clínicos electrónicos: revisión sistemática de la literatura. Gac Sanit. 2012; 26(5): 463-468.

18. Suárez-Obando F, Ordóńez A. Aspectos éticos de la informática médica: principios de uso y usuario apropiado de sistemas computacionales en la atención clínica. Acta Bioethica 2012; 18(2): 199-208.

19. Ministerio de Relaciones Exteriores. Decreto 830. Promulga Convención de los Derechos del Niño. (Publicado en el Diario Oficial el 27 de septiembre de 1990). Disponible en https://www.leychile.cl/N?i=15824\&f=1990-09-27\&p=.

20. Colegio Médico de Chile A.G.. Código de Ética; 2013. Disponible en http://www.colegiomedico.cl/wp-content/ uploads/2016/09/Codigo-de-Etica-Colegio-Medico-Chile-2013.pdf.

21. MINSAL. Decreto 158. Reemplaza DS 712/99. Aprueba reglamento sobre notificación de enfermedades transmisibles de declaración obligatoria. (Publicado en el Diario Oficial el 10 de mayo del 2005). Disponible en http://www.leychile. $\mathrm{cl} / \mathrm{N} ? \mathrm{i}=237770 \& \mathrm{f}=2010-04-19 \& \mathrm{p}=$.

22. Comisión Ministerial de Investigación en Salud. Acceso excepcional a la ficha clínica sin consentimiento informado, con fines de investigación. Análisis normativo: Ético y jurídico. Agosto 2015, Disponible en http://cei.usach.cl/wp-content/ uploads/2016/04/Ficha_Clinica.pdf.

23. Ministerio de Salud; Subsecretaría de Salud Pública. Ley 20.120. Sobre la investigación científica en el ser humano, su genoma, y prohíbe la clonación humana. (Publicado en el Diario Oficial el 22 de septiembre del 2006). Disponible en https://www.leychile.cl/N?i=253478\&f=2006-09-22\&p=.

24. Ramos S. La historia clínica en salud mental. Acta Bioethica 2015; 21(2): 259-268.

Recibido: 9 de agosto de 2017

Aceptado: 18 de mayo de 2018 